\title{
Note on Predictor-Corrector Formulas
}

The various predictor-corrector formulas for solving differential equations have considerable appeal because of their local control of truncation error and machine error. Of course, there is an expense for this control in terms of additional computing, so that a different method might be chosen if the accuracy does not need to be examined at each step. However, predictor-corrector formulas are quite generally used [1], [2].

There seems to be a natural tendency to repeat the "correction" formula, just like you take two aspirin instead of one. In fact the convergence of the iterative procedure defined by a "correction" formula has been studied, but evidently without inquiring whether the successive steps in the iteration actually afford an improvement. The purpose of this note is to point out that repeating a "correction" formula may worsen the result rather than improve it.

Consider $y^{\prime}=f(x, y)$, initial value $\left(x_{0}, y_{0}\right)$, predictor $y^{p}{ }_{n+1}=y_{n-1}+2 h f\left(x_{n}, y_{n}\right)$, and corrector $y_{n+1}^{c}=y_{n}+(h / 2)\left[f\left(x_{n}, y_{n}\right)+f\left(x_{n+1}, y^{p} n+1\right)\right]$.

This is a commonly used method with truncation errors $E^{p}=\left(h^{3} / 3\right) y^{\prime \prime \prime}$, $E^{c}=-\left(h^{3} / 12\right) y^{\prime \prime \prime} ; h$ is the increment in $x$, and $y^{\prime \prime \prime}$ denotes an appropriate mean value of $y^{\prime \prime \prime}(x)$. If $y^{\prime \prime \prime}(x)>0$ near the point in question and $y_{n+1}$ is the theoretical value we are seeking, then the signs of $E^{p}$ and $E^{c}$ show that $y_{n+1}^{p}-y_{n+1}<0$ while $y^{c}{ }_{n+1}-y_{n+1}>0$; also $y^{c}{ }_{n+1}>y^{p}{ }_{n+1}$.

Now suppose $\partial f / \partial y>0$ near the point in question: then applying the "corrector" to the "corrected" point gives

$$
y_{n+1}^{c c}=y_{n}+(h / 2)\left[f\left(x_{n}, y_{n}\right)+f\left(x_{n+1}, y_{n+1}^{c}\right)\right]>y_{n+1}^{c}>y_{n+1},
$$

so that $y_{n+1}^{c c}$ is farther than $y_{n+1}^{c}$ from $y_{n+1}$. It is also easy to give a geometric illustration for this result.

Criteria could be given indicating when repetition of the "corrector" formula will worsen and when it will improve $y^{c}{ }_{n+1}$. The situation is similar with other predictor-corrector formulas, and improvement is only a 50-50 proposition in that it hinges on the sign of a derivative. However, it seems much more practical to never repeat a "corrector," and if $\left|y_{n+1}^{c}-y_{n+1}^{p}\right|$ is too large to accept, then a smaller value of $h$ should be used.

IBM Corporation

D. D. WALL

Los Angeles, California

1. F. B. Hildebrand, Introduction to Numerical Analysis, McGraw-Hill, New York, 1956.

2. W. E. Milne, Numerical Solution of Differential Equations, John Wiley and Sons, New York, 1953.

\section{The Order of an Iteration Formula}

The order of an iteration formula indicates the rate of convergence of the iteration [1]. For example, Newton's formula $x_{n+1}=x_{n}-f\left(x_{n}\right) / f^{\prime}\left(x_{n}\right)$, for solving $f(x)=0$, is of order 2 since the number of correct decimal places approxi$x_{n+1}-\alpha=\left(x_{n}-\alpha\right)^{2} f^{\prime \prime}(\alpha) / 2 f^{\prime}(\alpha)+\theta$, where $\theta$ consists of terms of degree three and higher in $\left(x_{m}-\alpha\right)$ and when $f(\alpha)=0$. More generally, for the iteration 